\title{
INTERAÇÃO P x Fe EM MUDAS DE MACADÂMIA ${ }^{1,2}$
}

\author{
PAULO CESAR LIMA MARROCOS ${ }^{3}$, HERMINIA EMILIA PRIETO MARTINEZ4, VICTOR HUGO ALVAREZ \\ VENEGAS $^{5}$, CLÁUDIO HORST BRUCKNER ${ }^{4}$, REINALDO BERTOLA CANTARUTTI ${ }^{5}$
}

\begin{abstract}
RESUMO - Com objetivo de avaliar a interação $\mathrm{P}$ x Fe e seus efeitos no crescimento de mudas de macadâmia, foi conduzido um experimento na Universidade Federal de Viçosa, em solução nutritiva. Sete concentrações de P e de Fe foram combinadas de acordo com a matriz experimental BoxBerard aumentada (3), modificada, constituindo 14 tratamentos, em cinco repetições. Concentrações de Fe na solução nutritiva variando entre 0,136 e 0,289 $\mathrm{mmol} \mathrm{L}^{-1}$ reduziram em até $33 \%$ os teores de $\mathrm{P}$ nas folhas inferiores de plantas de macadâmia. Os teores de P nas folhas, nos tratamentos com a menor concentração de $\mathrm{P}$ e com concentrações de Fe variando entre 0,015 e $0,289 \mathrm{mmol} \mathrm{L}^{-1}$, mantiveram-se superiores às adequadas. Em solução nutritiva, a concentração de $\mathrm{P}$ pode ser menor que $0,03 \mathrm{mmol} \mathrm{L}^{-1}$. Os teores de Fe nas folhas de macadâmia aumentaram com a elevação da concentração de Fe na solução nutritiva e não foram afetados pela concentração de $\mathrm{P}$. No espaço experimental explorado a relação Fe $(\mathrm{mg} \mathrm{kg})^{-1}: \mathrm{P}(\mathrm{g} \mathrm{kg})^{-1}$ foi menor que 60, sugerindo que as folhas das plantas estariam com deficiência de Fe.
\end{abstract}

Termos para indexação: macadâmia, nutrição, relação fósforo/ferro.

\section{INTERACTION P X Fe IN SEEDLINGS OF MACADAMIA}

ABSTRACT - With the objective of evaluating the interaction $\mathrm{P} x \mathrm{Fe}$ and its effects on macadamia mute persons growth, an experiment was driven at the Federal University of Viçosa. Seven concentrations of $\mathrm{P}$ and Fe were combined in agreement with the experimental head office increased BoxBerard (3), modified, constituting 14 treatments, in five repetitions. Fe concentrations ranging from 0,136 and $0,289 \mathrm{mmol} \mathrm{L}^{-1}$ in the nutritious solution reduced the $\mathrm{P}$ concentrations in the lower leaves of macadamia plants. Plants growing with the lowest $\mathrm{P}$ concentration and with Fe concentration ranging from 0,015 and $0,289 \mathrm{mmol} \mathrm{L}^{-1}$ presented $\mathrm{P}$ concentrations superior to the adequate ones on the leaves. In nutritious solution, the $\mathrm{P}$ concentration can be lower than $0,03 \mathrm{mmol} \mathrm{L}^{-1}$. The increasing of Fe concentrations in macadamia leaves increased with the increasing of Fe concentration in the solution as well as they were not affected by $\mathrm{P}$ concentration increasing in the nutritious solution. In the whole experimental area, the rate of $\mathrm{Fe} \mathrm{mg} \mathrm{kg}{ }^{-1}: \mathrm{P} \mathrm{g} \mathrm{kg}^{-1}$ was lower than 60, suggesting that the leaves of those plants would be Fe-deficient.

Index terms: macadamia, nutrition, relationship phosphorus/iron.

\section{INTRODUÇÃO}

A macadâmia, uma das mais novas alternativas agrícolas do País, produz uma noz de alto valor no mercado internacional, com grande aceitação entres os consumidores. No Havaí, onde o cultivo desenvolveu-se em grande escala, são vários os problemas enfrentados pelos agricultores, entre eles a clorose de $\mathrm{Fe}$ induzida pelo excesso de $\mathrm{P}$.

A clorose das folhas de plântulas de macadâmia está associada à baixa reserva de Fe na semente (Guest, 1942). Fox \& Warner (1971) relatam que o excesso de $\mathrm{P}$ pode induzir o desenvolvimento de clorose em folhas de macadâmia, principalmente as novas. Eles concluíram que o problema é complexo e envolve interações entre a planta e o solo e entre o $\mathrm{P}$ e alguns micronutrientes, especialmente Fe e $\mathrm{Zn}$.

Jones et al. (1972) observaram que o alto teor de P na proximidade da raiz pode imobilizar o $\mathrm{Fe}$, limitando seu suprimento às raízes de macadâmia. Adams (1980) concluiu que a interação P x Fe é regulada geneticamente, podendo se manifestar em etapas separadas durante os processos de absorção, de translocação e de assimilação. Essa interação pode ser explicada pela reação de precipitação do $\mathrm{Fe}^{+3}$ com o fosfato, quer na zona externa à raiz, quer internamente, nos espaços intercelulares.

Para Gilfillan \& Jones (1968), quando o teor de P na folha excede 2,0 $\mathrm{g} \mathrm{kg}^{-1}$, com o teor de Fe em torno de $30 \mathrm{mg} \mathrm{kg}^{-1}$, manifesta-se a clorose. Hue et al. (1988) concluíram que a macadâmia produz bem quando o teor de P na folha está entre 1,0 e 1,4 $\mathrm{g} \mathrm{kg}^{-1}$, correspondendo a 0,03 e $0,05 \mathrm{mg} \mathrm{L}^{-1}$ na solução dos solos do Havaí. Teores acima dos mencionados resultavam em manifestação da clorose e redução na colheita. Para Nagao \& Hirae (1992), concentrações de 1,7 a 1,8 g kg ${ }^{-1}$ de P na folha já podem induzir os sintomas.

A deficiência de Fe pode ser resultado da insuficiência de Fe na planta ou ineficiência da planta em utilizar o Fe, e a clorose, em macadâmia, é resultado do uso ineficiente do Fe e não propriamente da deficiência (Fox \& Warner, 1971).
Handreck (1992) verificou uma tendência de redução no teor de $\mathrm{P}$ na folha e no caule de macadâmia com o incremento das doses de Fe, quando a fonte de $\mathrm{Fe}$ era o $\mathrm{FeSO}_{4} \cdot 7 \mathrm{H}_{2} \mathrm{O}$. Em contraste, o FeEDDHA não reduziu sistematicamente o teor de $\mathrm{P}$ na parte aérea. Concluiu que o sulfato ferroso e outras fontes de Fe capazes de promover altas concentrações de Fe na solução do solo parecem reverter os efeitos provocados por teores altos de $\mathrm{P}$.

Este experimento teve como objetivo avaliar a interação $\mathrm{P}$ x Fe e seus efeitos no crescimento de mudas de macadâmia, bem como de estabelecer os níveis críticos de $\mathrm{Pe}$ Fe nas folhas de plantas de macadâmia, em solução nutritiva.

\section{MATERIALEMÉTODOS}

O experimento foi instalado em casa-de-vegetação da Universidade Federal de Viçosa, com o cultivar Keauhou (HAES 246), de Macadamia integrifolia. Sementes foram colocadas para germinar em bandejas plásticas contendo areia lavada e as plântulas recém-germinadas foram submetidas a um período de adaptação de 30 dias em vasos contendo 13 litros de solução nutritiva a $1 / 2$ de força iônica da concentração adequada para a macadâmia. As concentrações de $\mathrm{P}_{-} \mathrm{H}_{2} \mathrm{PO}_{4}^{-}, \mathrm{N}_{-} \mathrm{NO}_{3}^{-}$ , $\mathrm{N}-\mathrm{NH}_{4}^{+}, \mathrm{K}^{+}, \mathrm{Ca}^{2+} \mathrm{Mg}^{2+}$ e S-SO ${ }_{4}^{2-}$ na solução integral foram de 0,$3 ; 14,0$; 1,$5 ; 4,0 ; 4,4 ; 1,0$ e $1,0 \mathrm{mmol} \mathrm{L}^{-1}$, respectivamente. As concentrações de micronutrientes foram estabelecidas de acordo com a solução de Clark (1975), exceto o Fe, usado na concentração de $0,076 \mathrm{mmol} \mathrm{L}^{-1}$ na forma de Fe-EDTA. Após período de adaptação, as plantas foram transferidas para vasos contendo 8 litros da solução nutritiva na concentração integral, com exceção para P e Fe. Sete concentrações de P e sete de Fe foram combinadas de acordo com a matriz experimental Box-Berard aumentada (3) e modificada, constituindo 14 tratamentos (Tabela 1), dispostos em delineamento de blocos ao acaso, com cinco repetições.

\footnotetext{
${ }^{1}$ (Trabalho 144/2002). recebido: 18/07/2002. Aceito para publicação: 28/05/2003.

${ }^{2}$ Parte da Tese apresentada, pelo primeiro autor, à Universidade Federal de Viçosa, para obtenção do título de Doctor Scientie.. O trabalho teve incentivo do CNPq durante o curso.

${ }^{3}$ Centro de Pesquisa do Cacau (CEPEC), CEPLAC, C.P. 07, 45.600-000, Itabuna (BA). E-mail: marrocos @ cepec.gov.br.

${ }^{4}$ Departamento de Fitotecnia, UFV, 36.571-000, Viçosa (MG).

${ }^{5}$ Departamento de Solos, UFV, 36.571-000, Viçosa (MG)
} 
TABELA 1 - Tratamentos estudados, obtidos pela matriz experimental Box-Berard aumentada (3) e modificada, com sete concentrações de $\mathrm{P}$ e sete de $\mathrm{Fe}$.

\begin{tabular}{ccccc}
\hline & \multicolumn{2}{c}{ Níveis } & \multicolumn{2}{c}{ Concentrações } \\
\cline { 2 - 5 } Tratamento & $\mathrm{P}$ & $\mathrm{Fe}$ & $\mathrm{P}$ & $\mathrm{Fe}$ \\
\hline & & & --------- & $\mathrm{mmol} \mathrm{L}^{-1}-------$ \\
1 & -1 & -1 & 0,150 & 0,076 \\
2 & -1 & 1 & 0,150 & 0,228 \\
3 & 1 & -1 & 0,450 & 0,076 \\
4 & 1 & 1 & 0,450 & 0,228 \\
5 & $-1,5$ & 0 & 0,075 & 0,152 \\
6 & 1,5 & 0 & 0,525 & 0,152 \\
7 & 0 & $-1,5$ & 0,300 & 0,038 \\
8 & 0 & 1,5 & 0,300 & 0,266 \\
9 & $-1,8$ & -1 & 0,030 & 0,076 \\
10 & -1 & $-1,8$ & 0,150 & 0,015 \\
11 & 1,8 & 1 & 0,570 & 0,228 \\
12 & 1 & 1,8 & 0,450 & 0,289 \\
13 & 0 & 0 & 0,300 & 0,152 \\
14 & $-1,8$ & $-1,8$ & 0,030 & 0,015 \\
\hline
\end{tabular}

As unidades experimentais foram constituídas por vasos contendo duas plantas. Durante o período experimental realizaram-se trocas periódicas das soluções, em intervalos determinados pela redução na condutividade elétrica das soluções a $30 \%$ do seu valor inicial. O volume de solução nutritiva nos vasos foi mantido pela reposição diária de água desionizada e o pH monitorado diariamente e mantido entre 5,0 e 5,5.

As plantas foram colhidas aos quarenta e quatro dias após a instalação do experimento, sendo subdivididas em folhas superiores, folhas inferiores, caules e raízes. Após lavagem em água desionizada, o material vegetal foi seco em estufa com circulação forçada de ar a $70^{\circ} \mathrm{C}$, até peso constante. A matéria seca foi pesada, sendo em seguida moída até passar em peneira de 20 mesh de abertura de malha. Para análise de $\mathrm{P}$ e de Fe, a matéria seca foi mineralizada pela mistura nítrico-perclórica (4:2 v/v). O P foi dosado colorimetricamente pelo método de redução do fosfomolibdato pela vitamina C, descrito por Braga \& Defelipo (1974), e o Fe determinado por espectrofotometria de absorção atômica.

Os dados obtidos foram submetidos à análise de variância e os efeitos de tratamentos avaliados por equações de regressão, ajustadas entre as variáveis avaliadas e as doses de $\mathrm{P}$ e de $\mathrm{Fe}$. As equações foram selecionadas em função da significância dos coeficientes de regressão, considerando o nível de significância até $10 \%$ de probabilidade e do maior coeficiente de determinação.

\section{RESULTADOSEDISCUSSÃO}

A produção de matéria seca de plantas de macadâmia (Tabela 2) não variou de forma significativa em função das concentrações de $\mathrm{P}$ e de $\mathrm{Fe}(\hat{\mathrm{Y}}=\overline{\mathrm{Y}}=$ média), não sendo possível estabelecer os níveis críticos de $\mathrm{P}$ e de Fe nas folhas. No entanto, as folhas das plantas de macadâmia crescidas nas soluções com concentrações maiores de $\mathrm{P}$ e menores de Fe e na solução com menor concentração de $\mathrm{P}$ e de Fe apresentaram sintomas típicos da deficiência de Fe, inicialmente uma clorose internerval (nervuras verdes sobre fundo amarelado) nas folhas novas, seguidas de intenso amarelecimento da lâmina foliar. Em um estágio mais avançado, observou-se necrose na ponta e nos bordos de algumas folhas, permanecendo verde apenas a nervura central.

Os teores de $\mathrm{P}$ nas folhas aumentaram linearmente com as concentrações de $\mathrm{P}$, mas decresceram com o incremento das concentrações de Fe (Tabelas 3 e 4). O maior teor de $\mathrm{P}$ nas folhas superiores foi observado na concentração de $\mathrm{Fe}$ de $0,119 \mathrm{mmol} \mathrm{L}^{-1}$, a partir daí, aumentos nas concentrações de Fe na solução provocaram redução nos teores de $P$ nas folhas, de aproximadamente $24 \%$ na menor concentração de $P$ e de aproximadamente $16,7 \%$ na maior concentração de P. Com a menor $\left(0,03 \mathrm{mmol} \mathrm{L}^{-1}\right)$ e a maior $\left(0,570 \mathrm{mmol} \mathrm{L}^{-1}\right)$ concentração de $\mathrm{P}$, os teores de $\mathrm{P}$ nas folhas superiores foram de 2,5 e 3,0 $\mathrm{g} \mathrm{kg}^{-1}$, respectivamente. Tais resultados sugerem que a influência do $\mathrm{Fe}$ na absorção do $\mathrm{P}$ pela planta é maior, em menores concentrações de P na solução.

TABELA 2 - Matéria seca de folha, caule, raiz, parte aérea (PA) e total de plantas de macadâmia, em função de combinações de $\mathrm{P}$ e de $\mathrm{Fe}$ em solução nutritiva

\begin{tabular}{|c|c|c|c|c|c|c|}
\hline \multicolumn{2}{|c|}{ Tratamento } & \multicolumn{5}{|c|}{ Matéria Seca } \\
\hline $\mathrm{P}$ & $\mathrm{Fe}$ & Folha & Caule & Raiz & PA & Total \\
\hline \multicolumn{2}{|c|}{----mmol L ${ }^{-1}----$} & & & g. planta & & ---'- \\
\hline 0,150 & 0,076 & 4,51 & 1,08 & 0,78 & 5,59 & 6,37 \\
\hline 0,150 & 0,228 & 4,67 & 0,92 & 0,77 & 5,58 & 6,35 \\
\hline 0,450 & 0,076 & 3,36 & 0,86 & 0,56 & 4,22 & 4,78 \\
\hline 0,450 & 0,228 & 3,86 & 0,85 & 0,51 & 4,71 & 5,21 \\
\hline 0,075 & 0,152 & 4,31 & 0,82 & 0,57 & 5,13 & 5,71 \\
\hline 0,525 & 0,152 & 3,45 & 0,85 & 0,55 & 4,30 & 4,86 \\
\hline 0,300 & 0,038 & 3,80 & 0,93 & 0,64 & 4,73 & 5,37 \\
\hline 0,300 & 0,266 & 5,08 & 1,21 & 0,96 & 6,29 & 7,25 \\
\hline 0,030 & 0,076 & 4,61 & 0,87 & 0,68 & 5,47 & 6,15 \\
\hline 0,150 & 0,015 & 4,29 & 0,91 & 0,75 & 5,20 & 5,95 \\
\hline 0,570 & 0,228 & 4,06 & 1,02 & 0,63 & 5,07 & 5,70 \\
\hline 0,450 & 0,289 & 3,69 & 0,82 & 0,51 & 4,51 & 5,02 \\
\hline 0,300 & 0,152 & 3,79 & 0,85 & 0,59 & 4,65 & 5,24 \\
\hline 0,030 & 0,015 & 3,09 & 0,75 & 0,55 & 3,84 & 4,39 \\
\hline
\end{tabular}

TABELA 3 - Teores de P e de Fe nos diversos compartimentos das mudas de macadâmia: folha superior (FS), folha inferior (FI), caule (CL) e raiz (RZ), em função das concentrações de $\mathrm{P}$ e de Fe aplicadas à solução nutritiva

\begin{tabular}{|c|c|c|c|c|c|c|c|c|c|}
\hline $\mathrm{P}$ & $\mathrm{Fe}$ & FS & FI & CL & RZ & FS & FI & CL & RZ \\
\hline \multicolumn{2}{|c|}{ Tratamento (mmol.L $\left.{ }^{-1}\right)$} & \multicolumn{4}{|c|}{ Teores de $\mathrm{P}\left(\mathrm{g} \mathrm{kg}^{-1}\right)$. } & \multicolumn{4}{|c|}{ Teores de $\mathrm{Fe}\left(\mathrm{mg} \mathrm{kg}^{-1}\right)$} \\
\hline 0,150 & 0,076 & 2,6 & 2,7 & 5,6 & 4,4 & 17,16 & 34,22 & 28,81 & 896,35 \\
\hline 0,150 & 0,228 & 2,3 & 2,4 & 4,9 & 5,2 & 21,25 & 44,17 & 31,41 & 811,57 \\
\hline 0,450 & 0,076 & 2,9 & 3,4 & 6,0 & 5,9 & 20,76 & 32,29 & 47,36 & 428,36 \\
\hline 0,450 & 0,228 & 2,9 & 3,4 & 4,9 & 5,6 & 22,62 & 42,85 & 33,72 & 549,82 \\
\hline 0,075 & 0,152 & 2,5 & 2,7 & 4,8 & 3,9 & 20,72 & 40,24 & 53,57 & 806,13 \\
\hline 0,525 & 0,152 & 3,0 & 3,5 & 6,3 & 6,1 & 23,92 & 33,09 & 55,96 & 773,93 \\
\hline 0,300 & 0,038 & 2,5 & 2,2 & 5,3 & 5,9 & 15,70 & 31,63 & 39,04 & 564,29 \\
\hline 0,300 & 0,266 & 2,5 & 2,5 & 5,2 & 5,1 & 25,95 & 46,05 & 31,13 & 684,12 \\
\hline 0,030 & 0,076 & 2,3 & 1,8 & 3,1 & 3,1 & 19,75 & 32,46 & 48,54 & 959,70 \\
\hline 0,150 & 0,015 & 2,6 & 2,5 & 5,0 & 4,8 & 16,27 & 33,84 & 38,95 & 975,83 \\
\hline 0,570 & 0,228 & 2,6 & 3,0 & 5,0 & 5,8 & 20,61 & 38,55 & 27,51 & 851,75 \\
\hline 0,450 & 0,289 & 2,2 & 2,3 & 4,6 & 5,1 & 20,44 & 38,86 & 63,75 & 551,15 \\
\hline 0,300 & 0,152 & 2,8 & 2,6 & 5,4 & 4,8 & 21,78 & 40,55 & 88,50 & 650,06 \\
\hline 0.030 & 0,015 & 2.2 & 1.9 & 3.8 & 3.9 & 16,50 & 29.67 & 40,03 & 694.85 \\
\hline
\end{tabular}


TABELA 4 - Resposta dos teores de P e de Fe nos diversos compartimentos das mudas (CM) de macadâmia: folha superior (FS), folha inferior (FI), caule (CL) e raiz (RZ), em função das concentrações de $\mathrm{P}$ e de $\mathrm{Fe}$ aplicadas à solução nutritiva

\begin{tabular}{|c|c|c|}
\hline \multicolumn{3}{|c|}{ Resposta dos teores de $\mathrm{P}$ às concentrações de $\mathrm{P}$ e de $\mathrm{Fe}$} \\
\hline $\mathrm{CM}$ & Equações de Regressão & $\mathrm{R}^{2}$ \\
\hline FS & $\hat{\mathrm{Y}}=0,2176+0,0991^{*} * \mathrm{P}+0,4491^{\circ} \mathrm{Fe}-1,8822 \mathrm{Fe}^{2}$ & 0,71 \\
\hline FI & $\hat{\mathrm{Y}}=0,1713+0,2095^{*} * \mathrm{P}+0,8800^{*} \mathrm{Fe}-3,2326 \mathrm{Fe}^{2}$ & 0,71 \\
\hline CL & $\hat{\mathrm{Y}}=0,3375+0,7974 * * \mathrm{P}+0,7065^{*} \mathrm{Fe}-3,377$ P.Fe & 0,72 \\
\hline $\mathrm{RZ}$ & $\hat{\mathrm{Y}}=0,3295+0,9409^{* *} \mathrm{P}-0,8626 * * \mathrm{P}^{2}$ & 0,96 \\
\hline $\mathrm{CM}$ & Resposta dos teores de $\mathrm{Fe}$ às concentrações de $\mathrm{P}$ e de $\mathrm{Fe}$ & $\mathrm{R}^{2}$ \\
\hline FS & $\hat{\mathrm{Y}}=14,3829+73,6238 * * \mathrm{Fe}-159,1160 * * \mathrm{Fe}^{2}$ & 0,75 \\
\hline FI & $\hat{\mathrm{Y}}=29,2787+77,2060^{* *} \mathrm{Fe}-109,7910 \mathrm{Fe}^{2}$ & 0,81 \\
\hline CL & $\hat{\mathrm{Y}}=17,8589+316,8280 * * \mathrm{Fe}-760,5580 * * \mathrm{Fe}^{2}$ & 0,96 \\
\hline
\end{tabular}

**Significativo a $1 \%$ de probabilidade.

*Significativo a $5 \%$ de probabilidade.

${ }^{\circ}$ Significativo a $10 \%$ de probabilidade.

O maior teor de $\mathrm{P}$ nas folhas inferiores observou-se na concentração de Fe de $0,136 \mathrm{mmol} \mathrm{L}^{-1}$. Na concentração de $\mathrm{P}$ de $0,03 \mathrm{mmol} \mathrm{L}^{-1}$, o teor de $\mathrm{P}$ foi de $2,4 \mathrm{~g} \mathrm{~kg}^{-1}$ e na concentração de $\mathrm{P}$ de $0,570 \mathrm{mmol} \mathrm{L}^{-1}$, o teor de $\mathrm{P}$ foi de $3,5 \mathrm{~g} \mathrm{~kg}^{-1}$. A maior concentração de Fe provocou redução no teor de $\mathrm{P}$ na folha, sendo esta redução maior na menor concentração de $\mathrm{P}$, de aproximadamente $33 \%$; já a redução no teor de $\mathrm{P}$ observada na maior concentração de $\mathrm{P}$ foi de $23 \%$. A redução nos teores de $\mathrm{P}$, como verificado, tanto nas folhas superiores como inferiores, concorda com o observado por Hue \& Nakamura (1988). Sendo as folhas inferiores de referência para o diagnóstico foliar da planta, vale considerar que o teor de $1,9 \mathrm{~g} \mathrm{~kg}^{-1}$ de $\mathrm{P}$ encontrado na menor concentração de $\mathrm{P}$ e de Fe é considerado alto, se comparado com os valores citados por Nagao \& Hirae (1992). Ele seria alto o suficiente para desenvolver os sintomas da clorose de $\mathrm{Fe}$ induzida por excesso de $\mathrm{P}$, dependendo do teor de $\mathrm{Fe}$ observado. Isso sugere que a dose de $\mathrm{P}$ pode ser ainda menor que a concentração de $0,03 \mathrm{mmol} \mathrm{L}^{-1}$ na solução nutritiva.

Os teores de $\mathrm{P}$ no caule aumentaram linearmente com as concentrações de $\mathrm{P}$ e $\mathrm{Fe}$, havendo ainda efeito negativo da interação entre as doses de $\mathrm{P}$ e de $\mathrm{Fe}$ (Tabelas 3 e 4). Nas menores concentrações de $\mathrm{P}$ e de $\mathrm{Fe}$, o teor de $\mathrm{P}$ foi de $3,7 \mathrm{~g} \mathrm{~kg}^{-1}$ na MS, com o aumento da concentração de $\mathrm{P}$; mantendo-se o Fe constante na menor concentração, houve incremento de aproximadamente $108 \%$ no teor de P. Mantendo-se constante a menor concentração de $\mathrm{P}$ e variando a concentração de Fe até a máxima, houve incremento no teor de $\mathrm{P}$ de aproximadamente $43 \%$. Entretanto, ao se elevarem as concentrações de $\mathrm{P}$ e de $\mathrm{Fe}$, há aumento no teor de $\mathrm{P}$ de apenas $19 \%$.

Os teores de $\mathrm{P}$ nas raízes (Tabelas 3 e 4) variaram em função das concentrações de $\mathrm{P}$ e não foram afetados pelas concentrações de Fe. Na menor concentração de $\mathrm{P}$, o teor de fósforo foi de $3,6 \mathrm{~g} \mathrm{~kg}^{-1}$ e na maior concentração de $\mathrm{P}$, o teor foi de $5,8 \mathrm{~g} \mathrm{~kg}^{-1}$, correspondendo a um incremento de aproximadamente $61 \%$.

Os teores de Fe nas folhas superiores e inferiores (Tabelas 3 e 4) aumentaram com as doses de $\mathrm{Fe}$, enquanto que não variaram com as doses de P. Na menor e maior concentração de Fe, os teores nas folhas superiores foram de 15,45 e de 22,37 $\mathrm{mg} \mathrm{kg}^{-1} \mathrm{de} \mathrm{Fe}$, respectivamente.

$\mathrm{Na}$ menor e na maior concentração de $\mathrm{Fe}$, os teores nas folhas inferiores foram de $28,8 \mathrm{mg} \mathrm{kg}^{-1} \mathrm{e}$ de $42,4 \mathrm{mg} \mathrm{kg}^{-1} \mathrm{de} \mathrm{Fe}$, respectivamente. Considerando que estas são as folhas indicadoras do estado nutricional da macadâmia, vale observar que os teores de Fe encontrados, mesmo na maior concentração de $\mathrm{Fe}$, são considerados baixos, se comparados ao teor de $50 \mathrm{mg} \mathrm{kg}^{-1}$ de $\mathrm{Fe}$, citado como adequado por Bittenbender \& Hirae (1990). As relações Fe:P obtidas foram menores que 60 , sugerindo que mesmo as folhas das plantas que permaneceram verdes estariam deficientes em Fe. Essa falta de expressão visual da deficiência pode caracterizar um estado de fome oculta ao elemento. Bittenbender \& Hirae (1990) citam ainda que a relação Fe $\mathrm{mg} \mathrm{kg}^{-1}: \mathrm{P} \mathrm{g} \mathrm{kg}$
${ }^{1}$ deve ser superior a 60 .

$\mathrm{O}$ teor de $\mathrm{Fe}$ no caule respondeu às concentrações de $\mathrm{Fe}$ e não foi afetado pelas concentrações de P. A concentração de Fe que proporcionou o maior teor no caule, $50,9 \mathrm{mg} \mathrm{kg}^{-1} \mathrm{de} \mathrm{Fe}$, foi de $0,208 \mathrm{mmol} \mathrm{\textrm {L } ^ { - 1 }}$. Na menor concentração de Fe, o teor foi de $22,4 \mathrm{mg} \mathrm{kg}^{-1} \mathrm{de} \mathrm{Fe}$, e na maior concentração, o teor foi de $48,3 \mathrm{mg} \mathrm{kg}^{-1}$ de Fe. Nas raízes, as médias dos teores de Fe variaram de 429,3 a 1021,7 $\mathrm{mg} \mathrm{kg}^{-1}$ de Fe.

\section{CONCLUSÕES}

Os teores de Fe nas folhas de macadâmia aumentaram com a elevação da concentração de Fe na solução nutritiva e não foram afetados pela concentração de $\mathrm{P}$ na solução nutritiva. $\mathrm{O}$ incremento das concentrações de Fe na solução nutritiva reduziu os teores de $\mathrm{P}$ nas folhas inferiores de plantas de macadâmia. As relações Fe mg kg-1 $: \mathrm{P} \mathrm{g} \mathrm{kg}^{-1}$ foram menores que 60 , sugerindo que mesmo as folhas das plantas que permaneceram verdes estavam deficientes em Fe. No período estudado, o crescimento das plantas não foi influenciado pelos teores de $\mathrm{P}$ e de Fe observados nas folhas.

\section{REFERÊNCIASBIBLIOGRÁFICAS}

ADAMS, F. Interaction of phosphorus with other elements in soils and in plant. In: KHASAWNEH, F.E.; SAMPLE, E.C.; KAMPRATH, E.J. (Ed.) The role of phosphorus in agriculture. Madison: ASA, CSSA, SSSA, 1980. p.655-680.

BITTENBENDER, H.C.; HIRAE, H.H. Common problems of macadamia nut in Hawaii. College of Tropical Agriculture \& Human Resources, University of Hawaii, 1990. 44p.

BRAGA, J.M.; DEFELIPO, B. Determinação espectrofotométrica de fósforo em extratos de solos e plantas. Revista Ceres, Viçosa, v.21, p.73-85, 1974.

CLARK, R.B. Characterization of phosphatase of intact maize roots. Journal of Agricultural and Food Chemistry, Washington, v. 23, p. 458-460, 1975

FOX, R.L.; WARNER, R.M. Excess de phosphate and micro-nutrient deficiency in macadamia. Hawaii Farm Science, Honolulu, v. 20, n. 4, p. 1-4, 1971.

GILFILLAN, J.M.; JONES, W.W. Effect of iron and manganese deficiency on the chlorophyll, aminoacid an organic acid status of leaves of macadamia. Proceeding of the American Society for Horticultural Science, v.93, n.4, p.210-14, 1968.

GUEST, P. The ralationship between chlorosis of macadamia seedlings and certain chemical constittuents of macadamia seeds. Proceeding of the American Society for Horticultural Science, Alexandria, v. 41, p. 61-64, 1942.

HANDRECK, K.A. Iron-phosphorus interactions inthe nutrition of seedling macadamia in organic potting media. Australian Journal of Experimental Agriculture, v.32, p.773-779, 1992.

HUE, N.V.; FOX, R.L.; McCALL, W.W. Chlorosis in macadamia as affected by phosphate fertilization and soil properties. Honolulu, Hawaii. Journal of Plant Nutrition, Monticello, v. 11, n. 2, p. 161-173, 1988.

HUE, N.V.; NAKAMURA, E.T. Iron chlorosis in macadamia as afected by phosphate-iron interactions. Journal of Plant Nutrition, Monticello, v. 11, n. 12, p. 1635-1648, 1988.

JONES, R.C.; SHIGEURA, G.T.; UEHARA, G. Microprobe investigation of phosphorus-induced chlorosis in macadamia. Hawaii Farm Science. Honolulu, v. 21, n. 9, p. 2-3, 1972.

MARSCHNER, H. Mineral nutrition of higher plants. London: Academic Press, 1995. 889p.

NAGAO, M.A.; HIRAE, H.H. Macadamia: cultivation e physiology. Critical Reviews in Plant Sciences, Boca Raton, v. 10, n. 5, p. 441-470, 1992.

TAIZ, L.; ZEIGER, E. Plant physiology. Redwood City: The Benjamin/ Cummings,. 1991.559p. 